\title{
Active Sensors for Health Monitoring of Aging Aerospace Structures
}

\author{
Victor Giurgiutiu \\ University of South Carolina, Mechanical Engineering Department, SC 29208, victorg@sc.edu \\ Jim Redmond, Dennis Roach, and Kirk Rackow \\ Sandia National Laboratories, Albuquerque NM, 87185-0847
}

\begin{abstract}
A project to develop non-intrusive active sensors that can be applied on existing aging aerospace structures for monitoring the onset and progress of structural damage (fatigue cracks and corrosion) is presented. The state of the art in active sensors structural health monitoring and damage detection is reviewed. Methods based on (a) elastic wave propagation and (b) electro-mechanical (E/M) impedance technique are cited and briefly discussed. The instrumentation of these specimens with piezoelectric active sensors is illustrated. The main detection strategies $(\mathrm{E} / \mathrm{M}$ impedance for local area detection and wave propagation for wide area interrogation) are discussed. The signal processing and damage interpretation algorithms are tuned to the specific structural interrogation method used. In the high-frequency $\mathrm{E} / \mathrm{M}$ impedance approach, pattern recognition methods are used to compare impedance signatures taken at various time intervals and to identify damage presence and progression from the change in these signatures. In the wave propagation approach, the acousto-ultrasonic methods identifying additional reflection generated from the damage site and changes in transmission velocity and phase are used. Both approaches benefit from the use of artificial intelligence neural networks algorithms that can extract damage features based on a learning process. Design and fabrication of a set of structural specimens representative of aging aerospace structures is presented. Three built-up specimens, (pristine, with cracks, and with corrosion damage) are used. The specimen instrumentation with active sensors fabricated at the University of South Carolina is illustrated. Preliminary results obtained with the E/M impedance method on pristine and cracked specimens are presented.
\end{abstract}

Keywords: piezoelectric sensors, active sensors, aging aircraft, damage detection, health monitoring, failure prevention, ultrasonics, pulse-echo detection, emitter-receptor detection, acousto-ultrasonic, signal analysis, wavelet transform.

\section{INTRODUCTION}

Health monitoring of aging structures is a major concern of the engineering community. This need is even more intense in the case of aging aerospace structures which have been operating well beyond their initial design life. Multi-site fatigue damage, hidden cracks in hard-to-reach locations, and corrosion are among the major flaws encountered in today's extensive fleet of aging aircraft and space vehicles. The durability and health monitoring of such structures form the subject of extensive research in many universities, government labs, and industry. This area is of growing concern and worthy of new and innovative approaches. The nation's safety and reliability record is excellent but the fatigue of its aging aerospace fleet is raising major concerns. Though well established design and maintenance procedures exist to detect structural fatigue, new and unexpected phenomena must be accommodated by the application of advanced flaw detection methods. One example is the case of the Aloha Airlines 1988 accident (Figure 1). This accident was due to a relatively new phenomenon, multi-site crack damage in the skin panel joints, resulting in catastrophic "un-zipping" of large fuselage panels. Subsequent analysis identified the multi-site crack damage phenomenon as a typical situation of damage synergism. The Aloha accident compelled the aerospace engineering community to take a fresh look at the fail-safe, safe-life, and damage tolerance design philosophies. The effect of aging on aircraft airworthiness and the deadly combination of fatigue and corrosion had to be reassessed. Prevention of such unexpected occurrences could be improved if on-board health monitoring systems exist that could assess the structural integrity and would be able to detect incipient damage before catastrophic failures occur To gain wide spread acceptance, such a system has to be cost effective, reliable, and compact/light weight. 


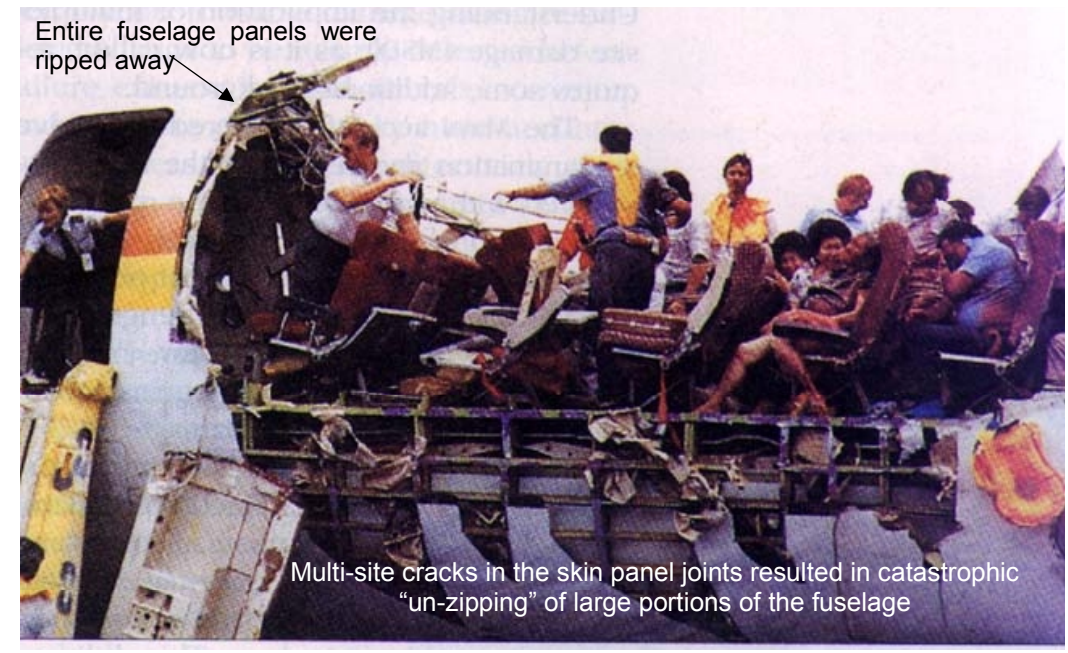

Figure 1 Aloha Airlines Boeing 737 accident on April 28, 1988 was due to multi-site crack damage in the skin panel joints resulting in catastrophic "un-zipping" of large portions of the fuselage.

Another important aspect related to the operation and maintenance of our aging aircraft fleet is that of cost. The United States spends more than $\$ 200$ billion each year on the maintenance of plant equipment and facilities. Aerospace maintenance and repairs represents about a quarter of a commercial fleet's operating costs (Figure 2 ). The mounting costs associated with the increasing needs of our aging infrastructure are rising at an unexpected rate. One of the main reasons for this rise in cost is that most of the inspections and structural health monitoring is performed manually. As aircraft age additional tasks such as Supplemental Structural Inspections are required. These increase the costs of maintaining an aging fleet. Select use of condition-based maintenance coupled with sensitive and continuous on-line monitoring of structural integrity would significantly reduce the cost of inspection programs. Retirement for cause instead of retirement as planned could reduce the cost while maintaining a safe operation life for many aging aircraft structures. The replacement of our present-day manual inspection with automatic health monitoring would substantially reduce the associated life-cycle costs. Hence, there is a need for reliable structural health monitoring systems that can automatically process data, assess structural condition, and

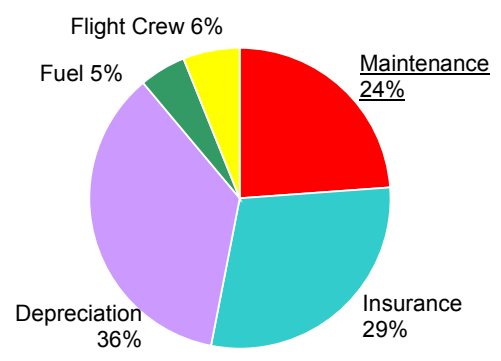

Figure 2 - Aircraft costs breakdown (after D. E. Good, AATD, US Army Aviation and Troop Command). signal the need for human intervention. Motivated by these pressing needs, considerable research efforts are being currently directed towards (a) development of new and better nondestructive inspection (NDI) techniques; (b) treating the aging fleet with fatigue life enhancement and life extension techniques; and (c) improving the inspection and maintenance procedures to better capture the unexpected occurrences (Bartkowicz, et al., 1996). Examples of (a) include thermography, digital radiography, scanning ultrasonics, eddy current, acoustic emission, Moire motion detection, shearography, and new holographic techniques. At the same time, a breakthrough in the approach can be achieved through (a) the development of health monitoring sensors; and (b) the construction of automated healthmonitoring systems. Among the available options for on-board structural health monitoring systems, the active-material sensors (active sensors) have the advantage of being slim and unobtrusive, readily integrated into structures, and selfexcited. Self excitation ensures that no cumbersome electrical excitation devices are required and that small-size electronics can be developed to accommodate the tight space and weight requirements of most aircraft structures. Health monitoring sensors based on active-material principles constitute an enabling technology of major interest (Boller, et al., 1999). Conventional passive sensors can only tell what happened to the structure, i.e., load and strain history. In contrast, active sensors should be able to interrogate the structure (e.g., through elastic waves) and find out "how it feels", i.e. the state of its health. Active sensors based on active-material principles (piezoelectricity, piezomagnetism, etc.) have emerged as prime candidates. Active sensors can act as both transmitters and receptors. As transmitters, active sensors generate elastic waves in the surrounding material. As receptors, they receive elastic waves and transform them into electric signals. It is conceivable to imagine arrays of active-sensors, in which each element would take, in turn, the role of transmitter and receptor, and thus scan large structural areas using ultrasonic waves. Alternatively, local-area impedance interrogation can be achieved by individual sensors, which are transmitters and receptors simultaneously. Active material sensors for structural health monitoring are conceptualized as thin piezoelectric wafer elements that can be either affixed (bonded) to existing structures, or incorporated (embedded) into new composite structures. The latter offer the possibility of multifunctional structural panels 
with integrated active-sensors and electronics (Noor, 1997). Such integrated structures could accommodate an automated health monitoring system that assesses the structure on a green-yellow-red scale, locates the damaged area, and tele-transmits a "structural health" bulletin to a central monitoring station for appropriate action (Figure 3).

A stand-alone sensory array system containing local area network, data logging, data evaluation, tele-transmission, and historical data storage and processing is envisioned. The development of this concept is essential for the commercial implementation of the proposed methodology. For deployment on existing aging structures, the health monitoring system must be self contained and autonomous. To achieve this, the active-sensors array will be connected with a local set of embedded electronics properly packaged to fit the into a restricted space envelop. After local data is read, interpreted and evaluated, the diagnostic is sent to the transmitter and uplinked into a data logger that monitors a large number of sites and critical structures. In this way, only the essential health-diagnostic data (condition GREEN, YELLOW, or RED) is transmitted to the structural-integrity data bank to be logged into the structural integrity reports of the critical structural systems. Such an autonomous health-monitoring system would be ideally suited for incipient damage detection, and would have wide use in aerospace, automotive, civil infrastructure and other industrial applications. As in other of today's engineering fields, the barrier to widespread industrial application of active-material based structural health monitoring is not in technology but in understanding. In spite of a host of experimental

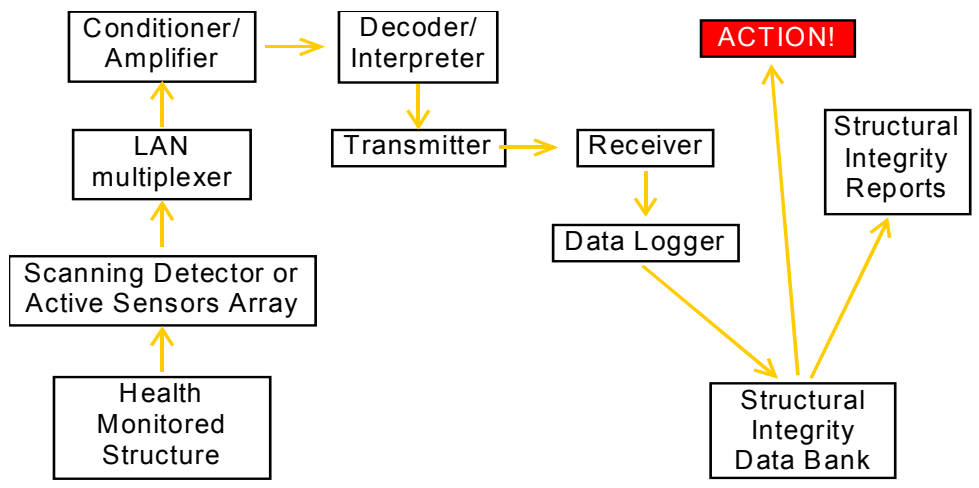

Figure 3 - General concept of a sensor-array structural integrity monitoring system. evidence, proof-of-concept demonstrations, and system planning, the understanding of the multidisciplinary phenomena associated with the interaction between the activematerial sensors and the host structure is still incomplete. Therefore, a concerted in-depth exploration of the basic principles is required. Theoretical modeling, performance prediction, laboratory experiments, and hypotheses testing, culminating with proof-of-concept demonstrations and pilot-plant experimentation are needed and should be planned. Indeed, our understanding of the use of active-sensors for health-monitoring is still incomplete, just as the understanding of ultrasonics was half a century ago. But active sensors have the potential to bring about a revolution in structural health monitoring, damage detection, and non-destructive evaluation just as significant as ultrasonic inspection did 50 years ago.

The present paper presents a project conducted in cooperation by the University of South Carolina and the Sandia National Laboratories Center for Airworthiness Assurance. The project sets forth to develop non-intrusive active sensors that can be applied on existing aging aerospace structures for monitoring the onset and progress of structural damage such as fatigue cracks and corrosion. This work in progress paper encompasses the sensor development, sensor deployment on representative aircraft structural specimens, structural interrogation strategies, and signal processing and damage interpretation algorithms. The active sensors under development are in the form of non-intrusive thin piezoelectric wafers of 5 to $10 \mathrm{~mm}$ square that can be easily attached to existing aging structures without changing the local and global structural dynamics. The structural interrogation strategies are two fold:

(a) For local area detection, the electro-mechanical (E/M) impedance method is applied to detect changes in the pointwise structural impedance resulting from the presence and propagation of structural damage.

(b) For large area detection, wave propagation techniques using Lamb and Love wave methods are used to identify zones in the monitored area that have undergone significant changes in their structural integrity.

Both methods utilize the same set of non-intrusive active sensors that are made to act as both E/M impedance transducers and emitters/receptors of elastic waves. The signal processing and damage interpretation algorithms are tuned to the specific structural interrogation method used. In the high-frequency E/M impedance approach, pattern recognition methods are used to compare impedance signatures taken at various time intervals and to identify damage presence and progression from the change in these signatures. In the Lamb/Love waves approach, the acousto-ultrasonic methods identifying changes in transmission velocity, phase, and additional reflection generated from the damage site are used. Both approaches benefit from the use of artificial intelligence neural networks algorithm that can extract damage features based on a learning process. To this purpose, structures both pristine and with known defects are used in our investigation. 


\section{STATE OF THE ART IN ACTIVE-SENSOR STRUCTURAL HEALTH MONITORING}

A large number of NDI techniques have been developed to identify local damage and detect incipient failure in aerospace structures. Among them, ultrasonic inspection based on elastic wave propagation, is well established and has been used in the engineering community for several decades (Krautkramer and Krautkramer, 1990). Also used is the mechanical impedance method (Cawley, 1984). The piezoelectric active-sensors methodology bears substantially on the experience accrued with these conventional ultrasonic techniques. However, major differences exist between conventional ultrasonics and activesensor methods. Drawbacks of the ultrasonic techniques are the bulkiness of transducers and the need for a normal (perpendicular) interface between the transducer and the test structure. The former limits the access of ultrasonic transducers to restricted spaces. The latter influences the type of waves that can be easily generated into the structure. In contrast with conventional ultrasonics, the active-sensors methods use wafer-like transducers that are permanently bonded to the structural surface. These active sensors are small, thin, unobtrusive, and non-invasive. They can be placed in very restrictive spaces, like in built-up aerospace structures. The surface bonded active sensors can easily produce waves traveling parallel to the surface and could detect damage that would escape an ultrasonic method. Additionally, the ultrasonic probes are moved across the structural surface through manual or semi-automated scanning, whereas embedded active sensors are permanently wired at predetermined locations. They can be remotely scanned through electronic switching.

(a)

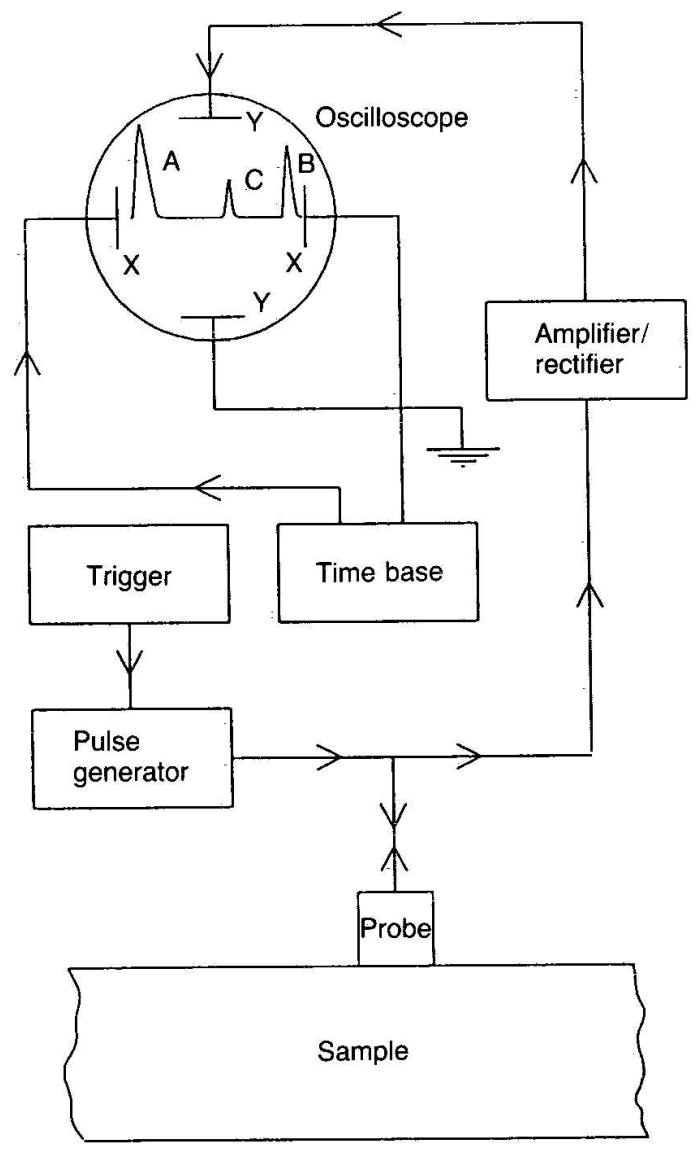

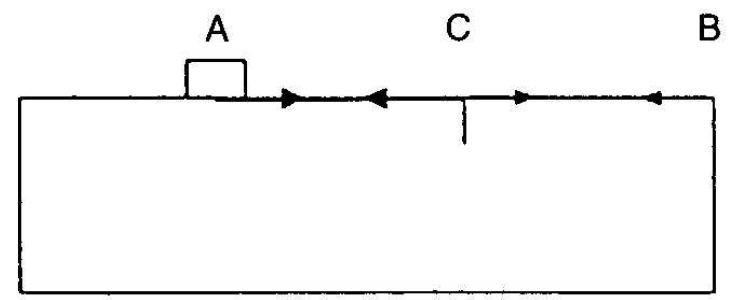

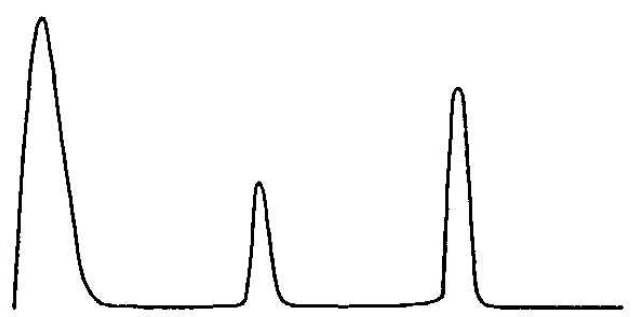

A
B

(b)

Figure 4 Ultrasonics damage detection techniques: (a) conventional pulse-echo method relies on normal waves to detect cracks parallel to the surface; (b) advanced methods using Rayleigh, Lamb, or Love waves are developed to detect cracks that lay perpendicular to the surface (Blitz and Simpson, 1996).

\subsection{Wave Propagation Methodologies}

Ultrasonic methods rely on elastic wave propagation and reflection within the material, and identify the field inhomogeneities due to local damage and flaws. Ultrasonic testing involves one or more of the following measurements: time of wave transit (or delay), path length, frequency, phase angle, amplitude, impedance, and angle of wave deflection (reflection and refraction). Conventional ultrasonic methods include the pulse-echo, the pulse-transmission (or shadow), and the pulse- 
resonance techniques (Blitz and Simpson, 1996). A piezoelectric ultrasonic probe placed on the structural surface induces ultrasonic waves in the material. Good contact between the probe and the structure is obtained by using special coupling gels. Depending on the incidence of the probe with respect to the structural surface, the waves created in the structures may be normal, shear, or a combination of the two. Normal waves are best suited for through-the-thickness detection. In the pulseecho method, defects are detected in the form of additional echoes (Figure 4a). In the pulse-transmission method, wave attenuation due to dispersed damage in the material is used as a flaw indicator. Since ultrasonic waves cannot be practically induced at right angles to the structural surface, localized surface flaws, and cracks with their plane perpendicular to the structural surface cannot be readily detected with conventional ultrasonic techniques. Advanced ultrasonic techniques rely on the generation, propagation, and detection of Rayleigh, Lamb, and Love waves (Viktorov, 1967) that act at the surface and in flexure mode. These waves may be generated with an angle probe, provided the probe angle is sufficiently large to trigger mode conversion (Figure 4b). Further advancements in this direction were achieved through acousto-ultrasonics (Duke, 1988). These techniques are now being transitioned to active sensors health monitoring.

Keilers and Chang (1995) identified delamination in composite beams using an array of PZT wafers affixed to a composite plate. Some of these PZT wafers acted as elastic wave generators, others acted as receptors of structural response. Modeling was performed with composite-beam bending and finite-element solutions. The PZT effect was introduced as (a) equivalent actuator moments; and (b) induced voltages. The detection of damage was deduced from the differences in structuralresponse magnitude over the $0-2 \mathrm{kHz}$ bandwidth. Other damage detection criteria, e.g., based on wave propagation, are also being studied (Chang, 1998). Moetakef et al. (1996) analyzed experimentally and numerically the capability of piezoceramic patches to generate elastic waves in beams and plates and discussed the possibility of using this method for damage detection. Lakshmanan and Pines (1997) used wave propagation to detect transverse cracks in a rotating composite beam from the scattering properties of the structure. This approach seemed to offer better resolution to detect high-frequency shifts due to transverse-crack damage. Blanas et al. (1998) studied the use of composites active sensors for acoustic-emission health monitoring. Kawiecki (1998) demonstrated experimentally the feasibility of nondestructive damage detection by an array of piezotransducers (25-mm square, $025 \mathrm{~mm}$ thick) bonded to the surface of four types of structures: aluminum beam; aluminum plate; concrete beam; concrete block. Jiang, Kabeya and Chonan (1999) studied the assessment of the location and characterization of damages by a longitudinal wave propagation measuring method. Two aluminum beams (1830 $\mathrm{mm} \times 12.7$ $\mathrm{mm} \times 3.18 \mathrm{~mm}$ and $940 \mathrm{~mm} \times 15 \mathrm{~mm} \times 2 \mathrm{~mm}$, respectively) were used. The first beam was instrumented with PZT wafer transducer pairs (top and bottom beam surfaces) at the ends of the beam and in the middle. Pulse-echo method was used on this beam. The transducer pairs placed at the end of the beam acted as transmitters and the pair in the middle of the beam acted as receiver. A small aluminum clamp was used to simulate damage. A sinusoidal burst transmitted from one of the ends was first received at the middle as direct transmission, and then was received again as reflection from the damage. The Daubechies ' $\mathrm{db} 8$ ' wavelet transform was used to process the signal. It showed some improvement over time-domain methods. The wave speed in the material, and the location of the damage could be determined. The second beam was instrumented with a PZT wafer pair at some place on its length, and with a third PZT wafer transducer at another location. The PZT wafer pair acted as a transmitter, while the third PZT wafer acted as a receiver. Damage was simulated with added mass (nut and bolt) and with a hole. Two methods (the difference method and the power consumption metric method) were used. In the difference method, the difference between the signals measured by the receiver in the pristine beam and in the "damaged" beam was computed. Initially the two signals were essentially identical, but clear differences arose upon arrival of the waves reflected from the damage. The time of arrival of the damage reflection was identified from the time when the difference between the two signals becomes significant. However, this method is not effective if the damage is located between the sensor and the actuator. In the power consumption metric method, the frequency spectrum of the time signal was computed using the FFT analysis. A so called "power consumption" value was calculated by summing the square of the spectral amplitudes. The power consumption metric was then computed by taking the difference of the pristine and damaged power consumption values and normalizing with the pristine value. Interesting results using wavelet transforms during active sensors structural health monitoring experiments were also presented by Deng, Wang, and Giurgiutiu (1999) and Lemistre et al. (1999).

\subsection{Local Impedance Methodologies}

The impedance method is a damage detection technique complementary to the wave propagation techniques. Ultrasonic equipment manufacturers offer, as options, mechanical impedance analysis (MIA) probes and equipment (Staveley NDT Technologies, 1998). The mechanical impedance method consists of exciting vibrations of bonded plates using a specialized transducer that simultaneously measures the applied normal force and the induced velocity. Cawley (1984) extended Lange's (1978) work on the mechanical impedance method and studied the identification of local disbonds in bonded plates using a small shaker. Though phase information was not used in Cawley's analysis, present day MIA methodology uses both magnitude and phase information to detect damage. 
The electro-mechanical (E/M) impedance method (Rogers and Giurgiutiu, 1997) is an emerging technology that offers distinctive advantage over the mechanical impedance method. While the mechanical impedance method uses normal force excitation, the E/M impedance method uses in-plane strain. While the mechanical impedance transducer measures mechanical quantities (force and velocity/acceleration) to indirectly calculate the mechanical impedance, the E/M impedance active sensor measures the $\mathrm{E} / \mathrm{M}$ impedance directly as an electrical quantity. The principles of the E/M impedance technique are illustrated in Figure 5. The effect of a piezo-electric active sensor affixed to the structure is to apply a local strain parallel to the surface that creates stationary elastic waves in the structure. The structure presents to the active sensor the drive-point impedance, $\quad Z_{s t r}(\omega)=i \omega m_{e}(\omega)+c_{e}(\omega)-i k_{e}(\omega) / \omega$.

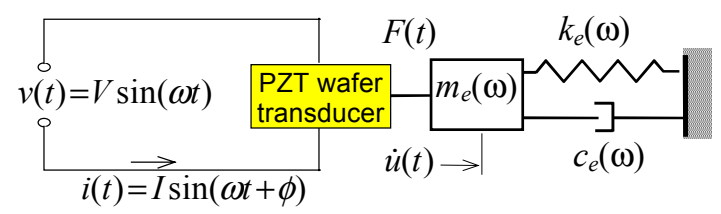

Figure 5 Electro-mechanical coupling between the PZT active sensor and the structure.

Through the mechanical coupling between the PZT active sensor and

the host structure, on one hand, and through the electro-mechanical transduction inside the PZT active sensor, on the other hand, the drive-point structural impedance gets directly represented in the effective electrical impedance as seen at the active sensor terminals. The apparent electro-mechanical impedance of the piezo-active sensor as coupled to the host structure is:

$$
Z(\omega)=\left[i \omega C\left(1-\kappa_{31}^{2} \frac{Z_{s t r}(\omega)}{Z_{P Z T}(\omega)+Z_{s t r}(\omega)}\right)\right]^{-1},
$$

where $Z(\omega)$ is the equivalent electro-mechanical admittance as seen at the PZT active sensor terminals, $C$ is the zero-load capacitance of the PZT active sensor, $\kappa_{31}$ is the electro-mechanical cross coupling coefficient of the PZT active sensor $\left(\kappa_{31}=d_{13} / \sqrt{\bar{s}_{11} \bar{\varepsilon}_{33}}\right), Z_{s t r}$ is the impedance of the structure, and $Z_{P Z T}$ is the impedance of the PZT active sensor. The electromechanical impedance method is applied by scanning a predetermined frequency range in the hundreds of $\mathrm{kHz}$ band and recording the complex impedance spectrum. By comparing the impedance spectra taken at various times during the service life of a structure, meaningful information can be extracted pertinent to structural degradation and the appearance of incipient damage. It must be noted that the frequency range must be high enough for the signal wavelength to be significantly smaller than the defect size.

Giurgiutiu and Rogers $(1997,1998)$ presented an extensive review of the state of the art in E/M impedance health monitoring of structures. Recent developments in this method focus on finding an effective damage metric to compare the E/M impedance spectra of pristine and damaged structures. Quin et al. (1999) developed an E/M impedance damage index (DI) scheme based on the differences of the piecewise integration of the frequency response curve between the damaged and undamaged cases. In addition, improved characterization of the structure is achieved by the separation of transverse and longitudinal outputs through directionally attached piezoelectrics (DAP). Lopes et al. (1999) used neural network techniques to process high-frequency E/M impedance spectra. In analytical simulation studies, a three level normalization scheme was applied to the E/M impedance spectrum base on the resonance frequencies. When applied to actual E/M experiments, the neural network approach was modified to another set of normalized values: (i) the area between damaged and undamaged impedance curves; (ii) the root mean square (RMS) of each curve; and (iii) the correlation coefficient between damaged and undamaged curves. These values were calculated for both real and imaginary parts of the impedance spectrum. Good identification of damage location and damage amplitude was reported.

\section{HEALTH MONITORING AND DAMAGE DETECTION STRATEGIES}

Our health monitoring approach simultaneously uses two major concepts for structural-interrogation and damage detection:

(a) Local-area sensing with the E/M impedance method, whereby each active sensor is excited independently and its impedance at very high frequencies $(100-1500 \mathrm{kHz}$, depending on feature size $)$ is measured. The real part of the E/M impedance reflects the state of structural health in the local area under the influence of the excited sensor. The integrity of the sensor itself is confirmed by the E/M impedance imaginary part.

(b) Wide-area sensing with wave propagation techniques whereby exciting an array of sensors in a round robin fashion and monitoring the features of the elastic wave transmission through the structure. General acoustoultrasonics methodology (Duke, 1988) was adapted to embedded active-material sensors format. Excitation at fixed frequency, frequency burst, or frequency sweep was attempted. The frequency band will be selected consistent with the size of the feature (defect or damage) that needs to be identified. 


\subsection{Damage Identification through E/M Impedance}

Consider an array of 4 active sensors as presented in Figure 6. Each active sensor has its own sensing area resulting from the application of the localization concept. This sensing area is characterized by a sensing radius and the corresponding sensing circle. Inside the sensing area, the sensor capability decreases as the distance from the sensor to the damage increases. A damage feature that is placed in the near field of the sensor is expected to create a larger disturbance in the sensor response than a damage feature placed in the far field. Effective area coverage is ensured when the sensing circles overlap. The diagnostics of the adjacent structure is performed using the active (real) part of the E/M impedance (Re Z). Incipient damage changes taking place in the structure are reflected in the drive-point structural impedance. Our experience has indicated that the change in the structural drive-point impedance extensively affects the real part of the effective electro-mechanical impedance of the piezo-electric active sensor affixed or embedded in the structure (Giurgiutiu and Rogers, 1998).
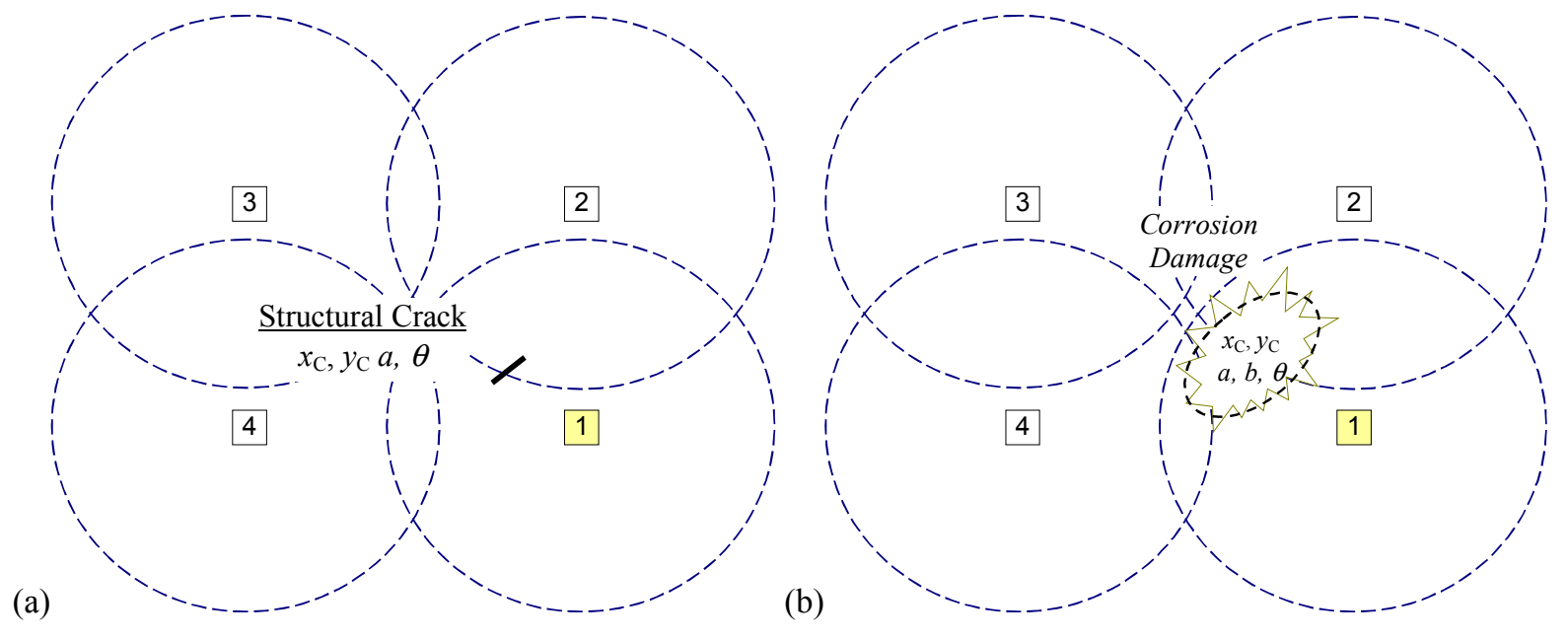

Figure 6 Damage detection using an array of 4 piezoelectric active sensors and E/M impedance method: (a) detection of structural cracks; (b) detection of corrosion damage. The circles represent the sensing radius of each active sensor.

\subsubsection{E/M Impedance Detection of Structural Cracks}

Figure $6 \mathrm{a}$ features a structural crack placed in the sensing circle of active sensor 1 . The crack presence modifies the structural field and effective drive-point structural impedance as seen by sensor 1. At the same time, the crack also belongs to the sensing circle of sensor 2, but it is right at the periphery of this circle. Thus, we expect that the effective drive-point structural impedance as seen by sensor 2 to also be affected, but to a much lesser extend than for sensor 1. Regarding sensors 3 and 4 , the structural crack is outside their sensing circles, hence their drive-point structural impedance will be almost unchanged. By virtue of Equation (1), these changes in the drive-point structural impedance will be directly reflected in the E/M impedance of the sensor. In conclusion, the crack illustrated in Figure 6a is expected to strongly modify the E/M impedance of sensor 1, to slightly modify that of 2 , and leave unchanged those of 3 and 4.

\subsubsection{E/M Impedance Detection of Corrosion Damage}

Figure $6 \mathrm{~b}$ features a patch of corrosion damage placed in the sensing circle of active sensor 1 . The corrosion damage also belongs to the sensing circles of sensors 2 and 4, but to a lesser extent. (For sensor 2, only half of the corrosion damage is inside its sensing circle; for sensor 4, the corrosion damage only touches the periphery of its sensing circle.) We expect that the effective drive-point structural impedance seen by sensor 1 will be strongly modified, that seen by sensor 2 will be modified to a lessor extent, and that of sensor 4 will be slightly modified. The drive-point impedance of sensor 3 will remain virtually unmodified. By virtue of Equation (2), these changes in the drive-point structural impedance will be directly reflected in the E/M impedance of the sensor. In conclusion, the corrosion damage (Figure 6b) is expected to strongly modify the E/M impedance of sensor 1, to somehow modify that of 2 , slightly modify that of 4 , and leave unchanged that of 3 .

\subsubsection{Active Sensor Self-diagnostics with the Electro-Mechanical Impedance Method}

Piezo-electric wafer transducers affixed to, or embedded into, the structure play a major role in the successful operation of the health monitoring and damage detection system. Integrity of the transducer and consistency of the transducer/structure 
interface are essential elements that can "make or break" an experiment. The general expectation is that, once the transducers have been placed on or into the structure, they will behave consistently throughout the duration of the health monitoring exercise. For real structures, the duration of the health monitoring exercise is extensive and can span several years. It also will encompass various service conditions and several loading cases. Therefore, in-situ self-diagnostics methods are mandatory. The transducer array should be scanned periodically as well as prior to any damage detection cycle. Active sensors integrity self-diagnostics methods based on the electro-mechanical (E/M) impedance technique can be easily achieved. The piezoelectric active sensor is predominantly a capacitive device that is dominated by its reactive impedance $1 / i \omega C$. Our preliminary tests have shown that the reactive (imaginary) part of the impedance $(\operatorname{Im} Z)$ can be a good indication of active sensor integrity. Base-line signatures taken at the beginning of endurance experiments when compared with recent reading could successfully identify defective active sensors.

\subsection{Damage Identification through Elastic Wave Propagation}

Consider an array of 4 active sensors as presented in Figure 8. Since piezoelectric active sensors can act as both sensors and actuators, our strategy assumes that one active sensor acts as actuator (1) while the others act as sensors $(2,3$, and 4). Active sensor 1 generates elastic waves that propagate through the material and are sensed at active sensors 2,3 , and 4 . The properties of these waves are affected by the presence of damage, and can be interpreted to yield damage location and amplitude. To maximize the amount of data and mitigate experimental error, a round-robin process is applied, whereby active sensors 2,3 , and 4 take, in turn, the function of wave generators, with the rest of the active sensors being wave receptors. This method can be applied to detect the two types of damage, cracks and corrosion.
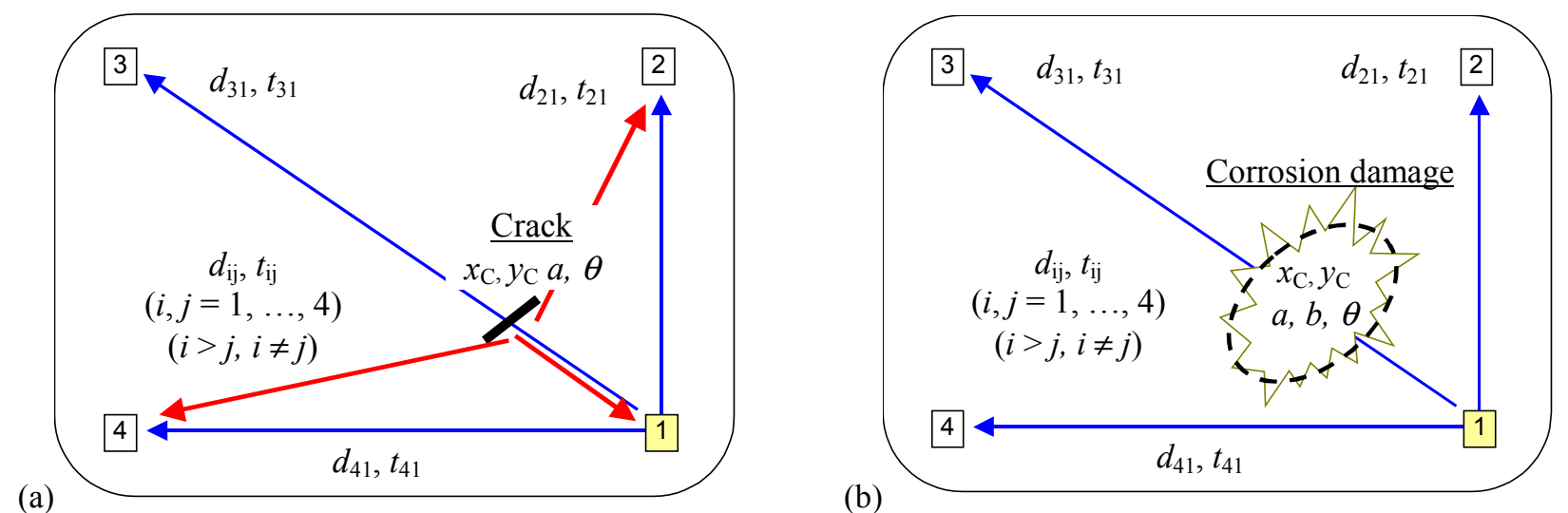

Figure 7 Damage detection strategy using an array of 4 piezoelectric active sensors and wave propagation techniques: (a) detection of structural cracks; (b) detection of corrosion damage

\subsubsection{Wave Propagation Detection of Structural Cracks}

Crack damage has to be characterized in terms of its location, $\left(x_{\mathrm{C}}, y_{\mathrm{C}}\right)$, and its size and orientation $(a, \theta)$. When a crack is present in the wave path, wave deflection, reflection, and transmission at the crack is expected to occur (Figure 7a). The proportion between deflection, reflection, and transmission will vary with damage size and orientation. In Figure 7a, active sensors 2 and 4 are shown to receive both direct and deflected waves. Active sensor 1 (the wave generator) also acts as a receptor and detects a reflected wave (echo). Active sensor 3 will receive a transmitted wave, its amplitude function of damage size. Thus, a matrix of valuable information in terms of event arrival time can be set up. Next, a round-robin procedure will be imposed, wherein active sensors 2, 3, and 4 will become, in turn, wave generators. In this way, further information will be obtained, and data error will be mitigated. Mathematical solution would yield the damage location, size and orientation $\left(x_{\mathrm{C}}, y_{\mathrm{C}}, a, \theta\right)$. In the solution algorithm, conventional linear algebra solution methods can be employed. Alternatively, neural network algorithms can be also used.

\subsubsection{Wave Propagation Detection of Corrosion Damage}

Unlike crack damage, which is 1-dimensional, corrosion damage is 2-dimensional and can cover a wide area. In Figure 7b, Active sensor 1 generates elastic waves that propagate through the material and are sensed at active sensors 2,3 , and 4 . The waves will propagate through damaged material different than through the pristine material. The difference will be in wave 
speed and attenuation. The corrosion damage has to be characterized in terms of its location, $\left(x_{\mathrm{C}}, y_{\mathrm{C}}\right)$ and its size and orientation ( $a, b, \theta$, i.e., the major and minor axes of a damage ellipse, and axis inclination). In Figure $7 \mathrm{~b}$, the waves received by the active sensors 2 and 4 are shown to travel through pristine material,and hence will not be affected. The waves received by active sensor 3 travel through damaged material, hence a modification in their travel time (wave speed) and attenuation is expected. (If damage is very intense, the material degradation may be very advanced, and it is possible that no waves are received at active sensor 3.) Next, a round-robin procedure will be imposed, wherein active sensors 2, 3, and 4 will become, in turn, wave generators. In this way, further information will be obtained, and data error will be mitigated. Mathematical solutions would yield the damage location, size and orientation $\left(x_{\mathrm{C}}, y_{\mathrm{C}}, a, b, \theta\right)$. In the solution algorithm, conventional linear algebra solutions or neural nets can be used.

\subsubsection{Selection and Optimization of Diagnostic Waves for Structural Damage Detection}

The type of waves used in the damage identification process can vary from conventional constant amplitude sines, to bursts, sweeps, impulse, etc. Four wave types are shown in Figure 8. The constant amplitude sine (Figure 8a) is the simplest waveform. However, the excitation frequency needs to be matched with the structural characteristics. The frequency sweep (Figure $8 \mathrm{~b}$ ) permits the excitation of more than one frequency in the same experiment. The impulse (Figure 8c) permits the excitation of a wide frequency spectrum, and can simulate impact damage events. Frequency burst (Figure 8d) is the excitation of choice for conventional ultrasonics. It contains a dominant frequency that can be tuned to the structural requirements. Since its frequency content is known, the burst wave can be readily detected and separated from background noise. Its limited duration facilitates the identification and analysis of burst reflection from defects and boundaries.

\subsection{Data Processing}

The information-rich data received from the sensors needs to be processed such that only its relevant content is retained. The elimination of spurious noise and far-field disturbances, and the identification of useful information directly related to structural damage has to be addressed through appropriate signal processing methodologies. Mathematically, the determination of the physical condition of a structure (pristine or damaged) using sensor measurements is a nonlinear inverse problem. Signal processing and interpretation methods can be application-specific (modelbased) or generic (non-model). In a continuing effort to satisfy a large class of applications, we are pursuing the generic signal-processing path. Our analysis utilizes conventional (Fourier analysis) and advanced (Wavelet analysis and digital filters) algorithms available in specialized software packages $\left(\right.$ Matlab $^{\mathrm{TM}}$, Labview ${ }^{\mathrm{TM}}$, Mathcad ${ }^{\mathrm{TM}}$, Autosignal $^{\mathrm{TM}}$, etc.). Artificial intelligence approaches will be utilized in the future. Neural networks and expert systems, which are complementary in scope and methodology, constitute the backbone of our data mining approach. The health monitoring methodology is based on historical data and spot-check results. Specific damage metrics are under development. (a) For local-area sensing, a metric comparing the historical

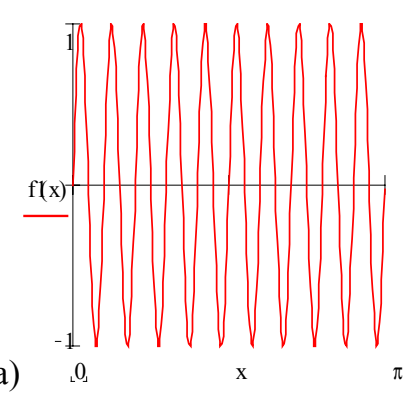

(b)

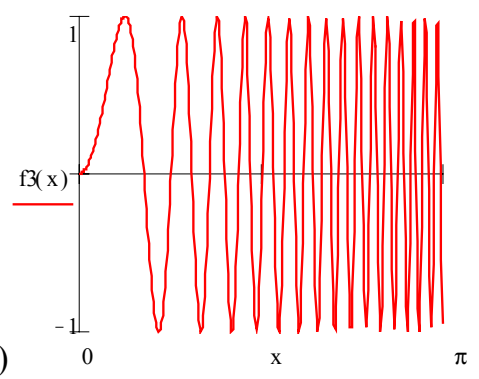

(c)

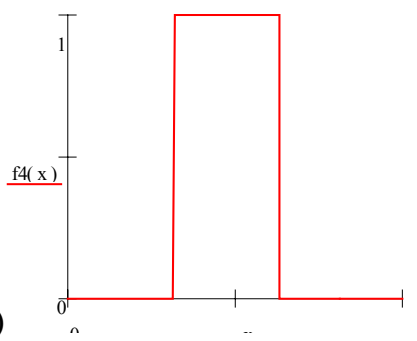

(d)

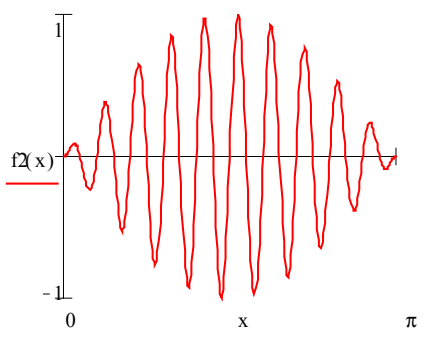

Figure 8 Diagnostic waves for active-sensor excitation: (a) constant amplitude sine; (b) frequency sweep; (c) impulse; (d) burst. impedance spectra will be used. RMS impedance change, piecewise integration of the frequency response curve, frequency shifts, and others indicators will be considered. (b) For wide-area sensing, several wave propagation techniques transitioned from ultrasonics and acoustoultrasonics methodology will be employed. Typical approaches include: pulse-echo; time of arrival; travel speed; attenuation; reflection; etc. 


\section{SPECIMEN DESIGN, FABRICATION, AND INSTRUMENTATION}

Realistic specimens representative of real-life aerospace structures with aging-induced damage (cracks and corrosion) were designed and fabricated. Figure 9 presents a blue print of the experimental panels developed at Sandia National Laboratories. The specimen has a built-up construction typical of conventional aircraft structures. It features a lap splice joint, tear straps, and hat-shaped stringer/stiffeners. The whole construction is made of 1-mm (0.040") thick 2024-T3 Al-clad sheet assembled with 4.2-mm (0.166") diameter countersunk rivets. Simulated cracks (EDM hairline cuts) and simulated corrosion damage (chem.-milled areas) were incorporated. Four specimens were constructed: (1) pristine; (2) with cracks only; (3) with corrosion only; (4) with a mix of cracks and corrosion. The specimens were instrumented with several piezoelectric wafer active sensors, 6-mm $(0.25$-in) square and $190 \mu \mathrm{m}(0.0075$-in) thick. The active sensors were fabricated in the USC Laboratory for Adaptive Materials and Smart Structures (LAMSS) from PZT (Lead Zirconate Titanate) single sheets supplied by Piezo Systems, Inc., Part \#T107-H4ENH-602, of 72-mm $\times 72$-mm $(2.85$-in $\times 2.85$-in). The PZT sheets were poled by the manufacturer to $1.9 \mathrm{kV} / \mathrm{mm}$ across the thickness. The active sensors were bonded to the panels with Micro Measurements, Inc. M-Bond 200 fast action glue, and instrumented with thin gage positive-connection leads. The negative connection was attached to the metallic panel specimen that acted as a common ground. Figure 10 shows a set of 4 such active sensors equidistantly placed in an row at right angle to a 0.75 " simulated crack (notch). Other sensors were placed in square patterns around other simulated defects. An impedance analyzer for the $\mathrm{E} / \mathrm{M}$ impedance testing, and wave generator, digital oscilloscope, pulser-receiver and $\mathrm{MHz}$ range $\mathrm{A} / \mathrm{D}$ boards for wave propagation testing were used in our experiments.

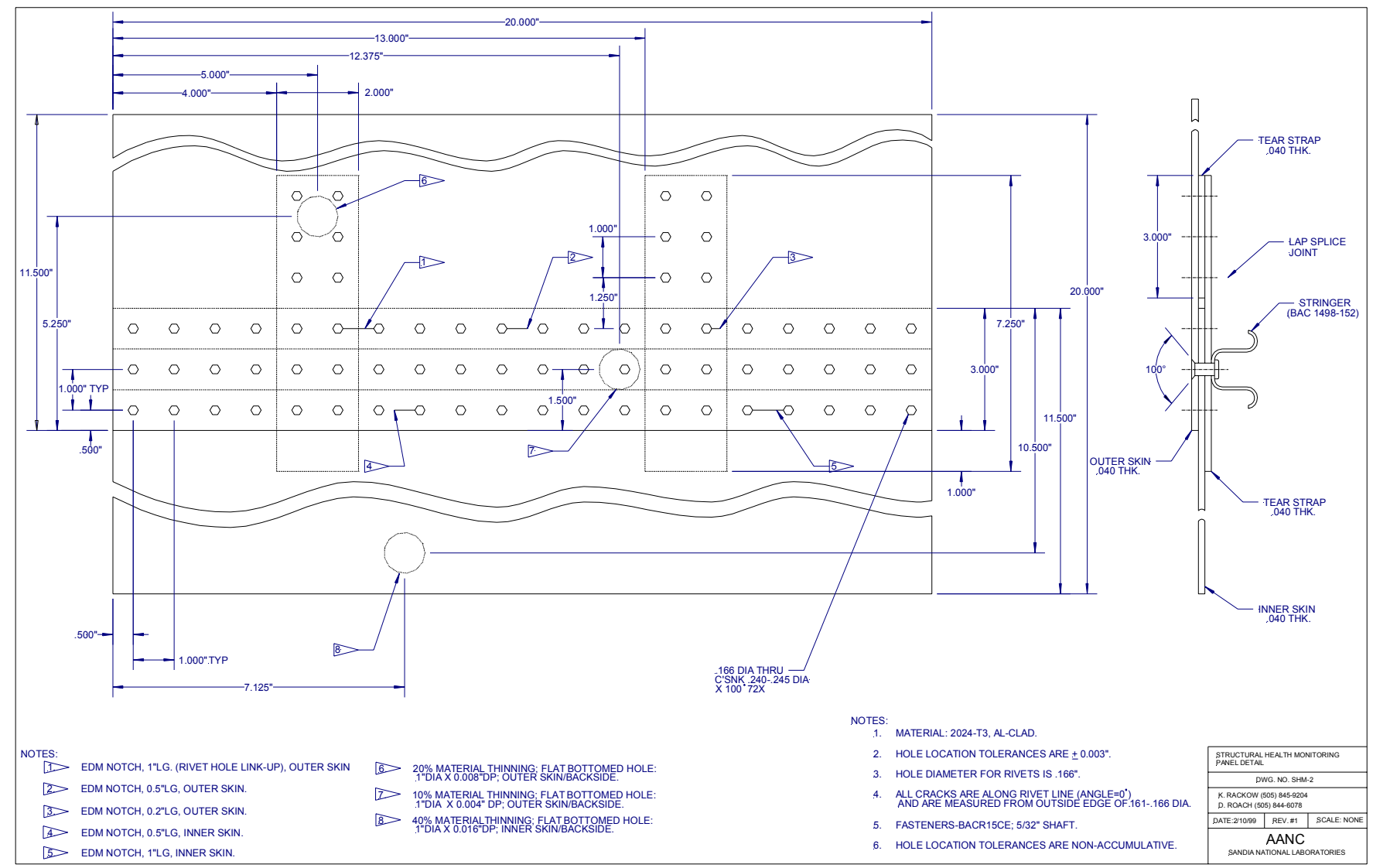

Figure 9 Blue print of the experimental panels developed at Sandia National Laboratories as specimens for testing active-sensor structural health monitoring, damage detection, and failure prevention methodologies. The specimen has a built-up construction typical of conventional aircraft structures. It contains simulated cracks (EDM hairline cuts) and simulated corrosion damage (chem.-milled areas). 


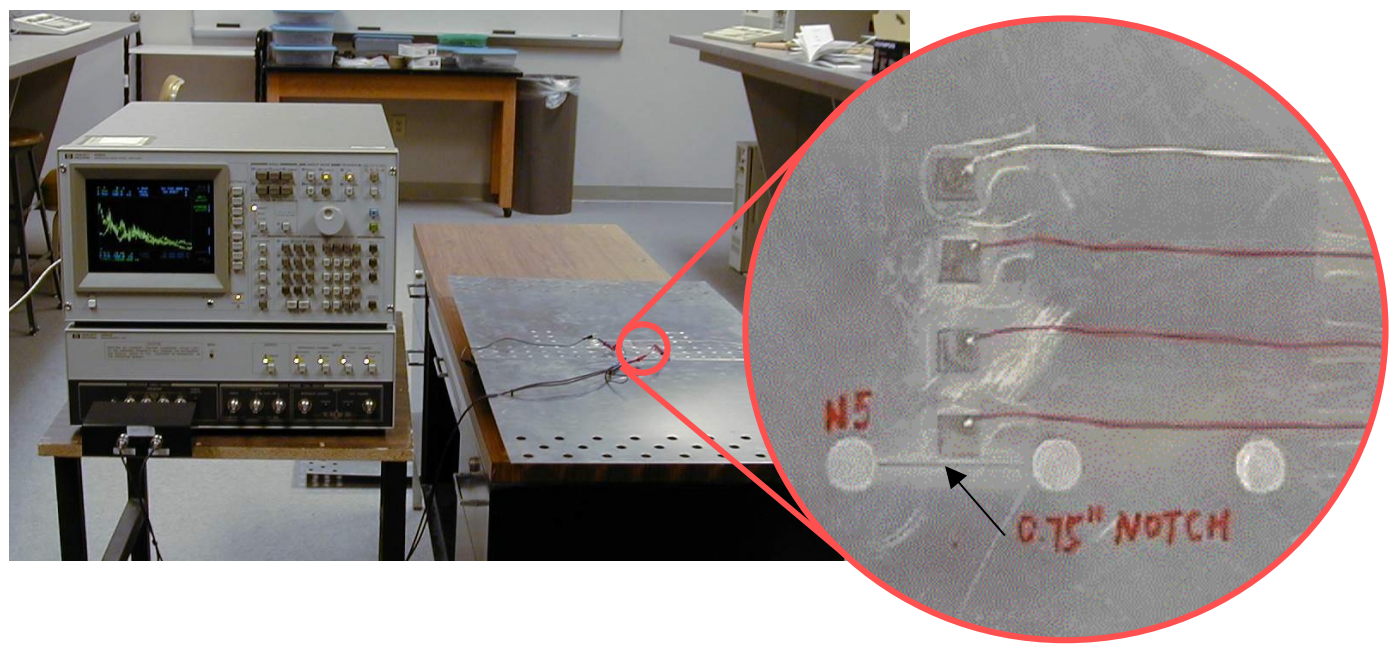

Figure 10 Active sensors instrumentation of the aerospace panel specimens containing simulated crack and corrosion damage. Three rivet heads, four PZT active sensors, and a 0.75" EDM-ed notch are featured in the photo insert. The full panel and the HP 4194A Impedance Analyzer are shown in the background.

\section{PRELIMINARY RESULTS}

Initial $\mathrm{E} / \mathrm{M}$ impedance measurements were made to detect cracks at the rivet location shown in Figure 10. Figure 11 shows the superposed results of E/M impedance testing performed on pristine (PZT-Ref) and cracked (PZT) specimens with the same sensor arrangement as shown in Figure 10. The sensors were equidistantly placed in a straight row along a path at right angles to the crack. It was expected that sensors further away from the crack would register less change in the E/M impedance spectrum than sensors closer to the crack. The sensor closest to the crack is expected to show the largest change in the $\mathrm{E} / \mathrm{M}$ impedance change. These presumptions are, to a certain extent, confirmed by the experimental results shown in Figure 11. However, other changes are also present in the $\mathrm{E} / \mathrm{M}$ impedance spectrum. The cause of these changes needs to be identified and explained. We are currently conducting experiments on standard $100 \mathrm{~mm}$ square aluminum sheet specimens in order to reduce and quantify the variability associated with sensor manufacturing and installation.

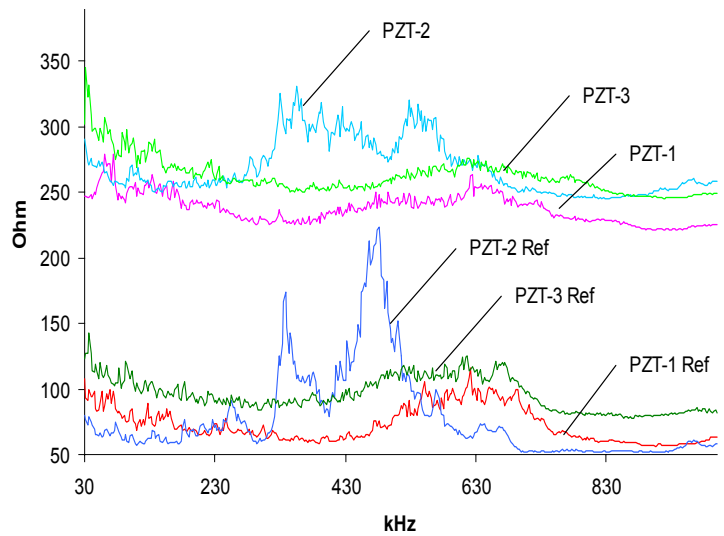

Figure 11 Preliminary E/M impedance results with active sensors placed on an aerospace panel specimens containing simulated crack and corrosion damage. (For graphing, the "damage" data was shifted up by $200 \Omega$.)

\section{CONCLUSION}

A project to develop non-intrusive active sensors that can be applied on existing aging aerospace structures for monitoring the onset and progress of structural damage (fatigue cracks and corrosion) has been presented. The state of the art in active sensors structural health monitoring and damage detection was reviewed. Methods based on (a) elastic wave propagation and (b) electro-mechanical $(\mathrm{E} / \mathrm{M})$ impedance technique were cited and briefly discussed. Damage detection strategies (E/M impedance for local area detection and wave propagation for wide area interrogation) were presented and discussed. It was noted that the signal processing and damage interpretation algorithms have to be tuned to the specific structural interrogation method. In the high-frequency E/M impedance approach, pattern recognition methods can be used to compare impedance signatures taken at various time intervals and to identify damage presence and progression from the change in these signatures. In the wave propagation approach, the acousto-ultrasonic methods identifying the reflection generated from the damage site and changes in transmission velocity and phase should be used. Both approaches benefit from the use of artificial intelligence neural networks algorithm that can extract damage features based on a learning process. Design and fabrication of structural specimens representative of aging aerospace structures with crack and corrosion damage was presented. The 
instrumentation of these specimens with piezoelectric active sensors was illustrated. To this purpose, structures both pristine and with known defects were used in the investigation. Preliminary results showing changes in the E/M impedance signatures with proximity of crack damage were presented. Much of the measured difference between the test specimens could be attributed to variations in sensor fabrication and installation, however. Therefore, further work is in progress to refine the sensor fabrication and installation procedures in order to improve the damage detection method's predictability and repeatability.

\section{ACKNOWLEDGMENTS}

The financial support of Department of Energy through the Sandia National Laboratories, contract doc. \# BF 0133 is thankfully acknowledged. Sandia National Laboratories is a multiprogram laboratory operated by Sandia Corporation, a Lockheed Martin Company, for the United States Department of Energy under contract DE-AC04-94AL85000. Thanks are expressed to graduate research assistants Andrei Zagrai and JingJing (Jack) Bao from the University of South Carolina for collecting some literature references and performing the preliminary experiments.

\section{REFERENCE}

Bartkowicz, T. J., Kim, H. M., Zimmerman, D. C., Weaver-Smith, S. (1996) “Autonomous Structural Health Monitoring System: A Demonstration", Proceedings of the 37th AIAA/ASME/ASCE/AHS/ASC Structures, Structural Dynamics, and Materials Conference, Salt-Lake City, UT, April 15-17, 1996

Blanas, P., Wenger, M. P., Rigas, E. J., and Das-Gupta, D. K. (1998) “Active Composite Materials as Sensing Element for Fiber Reinforced Smart Composite Structures", Proceedings of the SPIE North American Conference on Smart Structures and Materials, SPIE Vol. 3329, San-Diego, CA, March 1-5, 1998.

Boller, C., Biemans, C., Staszewski, W., Worden, K., and Tomlinson, G. (1999) "Structural Damage Monitoring Based on an ActuatorSensor System", Proceedings of SPIE Smart Structures and Integrated Systems Conference, Newport CA. March 1-4, 1999

Cawley, P., 1984, "The Impedance Method for Non-Destructive Inspection", NDT International, Vol. 17, No. 2, pp. 59-65.

Cawley, P. (1997) "Quick Inspection of Large Structures Using Low Frequency Ultrasound”, Structural Health Monitoring - Current Status and Perspective, Fu-Kuo Chang (Ed.), Technomic, Inc., 1997.

Chang, F.-K. (1998) "Manufacturing and Design of Built-in Diagnostics for Composite Structures", 52 $2^{\text {nd }}$ Meeting of the Society for Machinery Failure Prevention Technology, Virginia Beach, VA, March 30 - April 3, 1998.

Deng, X., Wang, Q., and Giurgiutiu, V. (1999) "Structural Health Monitoring Using Active Sensors and Wavelet Transforms", Paper \# 3667-35, SPIE's $6^{\text {th }}$ Annual International Symposium on Smart Structures and Materials, 1-5 March 1999, Newport Beach, CA

Duke, J. C. Jr., Acousto-Ultrasonics - Theory and Applications, Plenum Press, 1988.

Giurgiutiu, V., and Rogers, C. A. (1997) " Electro-Mechanical (E/M) Impedance Method for Structural Health Monitoring an NonDestructive Evaluation", Int. Workshop on Structural Health Monitoring, Stanford University, CA, Sep. 18-20, 1997, pp. 433-444

Giurgiutiu, V., and Rogers, C. A. (1998) "Recent Advancements in the Electro-Mechanical (E/M) Impedance Method for Structural Health Monitoring and NDE, Proceedings of the SPIE's 5th International Symposium on Smart Structures and Materials, 1-5 March 1998, Catamaran Resort Hotel, San Diego, CA, SPIE Vol. 3329, pp. 536-547

Kawiecki, G. (1998) "Piezogenerated Elastic Waves for Structural Health Monitoring", Proceedings of SMART-98, NATO Advanced Research Workshop, June 16-19, Pultusk, Poland

Keilers, C. H., Chang, F.-K. (1995) "Identifying Delaminations in Composite Beams Using Built-in Piezoelectrics: Part I - Experiments and Analysis; Part II An Identification Method", Journal of Intelligent Material Systems and Structures, Vol. 6, pp. 649-672, September, 1995.

Lakshmanan, K. A. and Pines, D. J. (1997) "Modeling Damage in Composite Rotorcraft Flexbeams Using Wave Mechanics", Journal of Smart Materials and Structures (in press)

Lopes Jr., V., Park, G., Cudney, H., and Inman, D., (1999) "Smart Structures Health Monitoring Using Artificial Neural Network", $2^{\text {nd }}$ International Workshop of Structural Health Monitoring, Stanford University, September 8-10, 1999, , pp. 976-985.

Moetakef, M., Joshi, S., and Lawrence, K., (1996) "Elastic Wave Generation by Piezoceramic Patches", AIAA Journal Vol. 34, No. 10. October 1996, pp. 2110-2117.

Noor, A. K., Venneri, S. L., Paul, D. B., and Chang, J. C. I., (1997) "New Structures for New Aerospace Systems", Aerospace America, November 1997, pp. 26-31.

Rogers, C. A. and Giurgiutiu, V. (1997) "Electro-Mechanical (E/M) Impedance Technique for Structural Health Monitoring and NonDestructive Evaluation", Invention Disclosure No. 97162, University of South Carolina Office of Technology Transfer, July 1997.

Viktorov, I. A., 1967, Rayleigh and Lamb Waves”, Plenum Press, New York, 1967

Krautkramer, Josef; Krautkramer, Herbert (1990) Ultrasonic Testing of Materials, Springer-Verlag, 1990.

Blitz, Jack; Simpson, Geoff (1996) Ultrasonic Methods of Non-Destructive Testing, Chapman \& Hall, 1996.

Lemistre, M.; Gouyon, R.; Kaczmarek, H.; Balageas, D. (1999) "Damage Localization in Composite Plates Using Wavelet Transform Processing on Lamb Wave Signals", $2^{\text {nd }}$ International Workshop of Structural Health Monitoring, Stanford University, September 810, 1999, pp. 861-870. 